\title{
Theoretical model and design of electroadhesive pad with interdigitated electrodes
}

\section{Changyong Cao ${ }^{1,2,3^{*}}$, Xiaoyu Sun ${ }^{3,4}$, Yuhui Fang ${ }^{1,2}$, Qinghua Qin ${ }^{1}$, Aibing $\mathrm{Yu}^{5}$ and Xi-Qiao Feng ${ }^{3}$}

${ }^{1}$ Research School of Engineering, Australian National University, Canberra, ACT 0200, Australia

${ }^{2}$ Department of Mechanical Engineering and Materials Science, Duke University, Durham, NC 27708, USA

${ }^{3}$ Institute of Biomechanics and Medical Engineering, Department of Engineering Mechanics, Tsinghua University, Beijing 100084, China

${ }^{4}$ Department of Engineering Mechanics, School of Civil Engineering, Wuhan University, Wuhan 430072, China

${ }^{5}$ Department of Chemical Engineering, Monash University, Clayton, VIC 3800, Australia.

* Corresponding author. Email: changyong.cao@ duke.edu, Tel: +1-919-681-9593, Fax: +1-919-660-5293.

\begin{abstract}
Electric fields alter adhesive forces between materials. Electroadhesive forces have been utilized in diverse applications ranging from climbing robots, electrostatic levitation to electro-sticky boards. However, the design of electroadhesive devices still largely relies on empirical or "trial-and-error" approaches. In this work, a theoretical model is presented to analyze the electrostatic field between the supporting wall and the electroadhesive device with periodic coplanar electrodes. The air-gap between the surface of electroadhesive device and the dielectric wall is explicitly taken into account in the model to consider its significant impact on electroadhesive forces. On the basis of this model, the electroadhesive force is calculated by using the Maxwell stress tensor. The effects of key design parameters and working environments on the electroadhesion behavior are further investigated. This study not only provides a tool to reveal the underlying mechanisms of electroadhesion but also suggests potential strategies to optimize novel electroadhesive devices for engineering applications.
\end{abstract}

Key words: theoretical model, electroadhesive pad, interdigitated electrodes, electroadhesion, dry adhesion, soft robotics.

\section{Introduction}

Electric fields can alter adhesive forces between materials, which allow surfaces to reversibly attach to each other without the use of mechanical grippers, suckors, fasteners, or chemical adhesives [1-6]. Electroadhesive devices have been developed by patterning electrodes in air or matrices of insulating dielectrics. Once an electric 
voltage is applied, the devices can invoke the adhesive forces to a wide variety of substrate materials (e.g., silicon, silicon dioxide, wood, drywall, glass, concrete, steel, and plastics) with various shapes, sizes, and roughness [2, 5-7]. Compared to mechanical and chemical adhesions, the electroadhesion has distinct advantages, e.g., fast response (response time $<10 \mathrm{~ms}$ ), quiet operation, low cost, easy control, low energy consumption, and dust tolerance [2]. Due to these many benefits, electroadhesive devices have been widely used in various applications. Examples include electrostatic levitation used in liquid-crystal-display manufacturing [4, 8-12], electroadhesive grippers for handling microcomponents of semiconductors $[13,14]$, and electroadhesive pads for wall climbing robots $[2,5,6$, $15,16]$ and sticky boards [17].

An electroadhesive device uses the electrostatic force between the supporting material (e.g., wall surface) and the electroadhesive pad. For Coulomb-type electrostatic pads (ESP), the electrostatic force is generated by the dielectric polarization due to the electric potential difference. Based on the charge (or electrode) configuration, ESPs can be classified into two types: mono-polar (plate-plate-capacitors) and bipolar (interdigitated electrodes) [1]. Mono-polar type ESPs generate adhesive forces in accordance with the principle that the two plates in a parallel capacitor attract each other if there is a voltage difference between them. Therefore, the target structure (e.g., a concrete wall) is required to be conductive such that a capacitor is formed between the electrode and the supporting wall [18]. A bipolar type electroadhesive device is typically constituted by interlacing the fingers of two conducting combs, as shown in Figure 1. The space between the electrode fingers is usually filled with an electrical insulator. The fingers and the filler are insulated from the substrate by a dielectric layer (i.e. the cover in Figure 1). When alternating positive and negative charges are induced on the adjacent electrodes and the device is placed in contact with a wall, the electric fields set up opposite charges on the wall and thus cause electrostatic adhesion between the electrodes and the induced charges on the supporting wall. This force may be explained by two mechanisms, namely, the gradient force and the Johnsen-Rahbeck force [19].

Despite these technologically important applications of electroadhesion, the design of electroadhesive devices has largely relied on trial-and-error approaches and the electroadhesive stresses and energy are usually estimated by empirical equations $[2,7,10,13,15]$. Prahlad and coworkers experimentally investigated the compliant electroadhesion technique and developed a variety of wall climbing robots such as tracked and legged robots [2]. They tested the attractive force of the robots climbing on distinct surfaces of materials and demonstrated the validity and superior performance of electroadhesion in climbing robots. Yamamoto et al. [15] designed a wall climbing robot with flexible electrodes that were fabricated by a plastic film and a conductive foil. They tested the attaching 
performance of the flexible electrode panel against both conductive and non-conductive walls, in which the adhesive forces is proportional to the square of voltage. Other innovative designs, e.g. gecko inspired electrostatic-chuck (ESC) [1], were also proposed to improve the poor performance of conventional ESCs on relatively rough surfaces. However, most of the studies were mainly experimental and there is still a lack of theoretical models to reveal the relationship between the adhesive force and the key design parameters. Optimal design principles are still unavailable for a majority of electroadhesive devices, in which the electrodes are actually embedded in insulating dielectrics. Only recently, Woo and Higuchi [9] developed a numerical model of electrostatic-levitation to calculate the forces generated by electrodes and discussed the optimization of the electric charging rate with respect to the geometrical parameters of electrodes.

In this paper, we attempt to theoretically address the basic design principles of electroadhesion. A semianalytical method is presented to calculate the electric field in a four-layer or five-layer model. The Maxwell stress tensor is used to calculate the desired electroadhesive force. We demonstrate that various key factors, including the dielectric constants of constituent materials, the thicknesses of cover layer, the air-gap, and the supporting walls, as well as the applied voltage and the dielectric constant of walls, have significant effect on the performance of electroadhesive devices. This work not only helps in the comprehension of the adhesion mechanisms in electrostatic devices but also supplies guidance for their optimal design.

\section{Model of Electroadhesive Forces}

Consider an electroadhesive pad consisting of periodic coplanar electrodes sandwiched between a dielectric cover and a substrate, as shown in Figure 1. Such electroadhesive pads can be made in a variety of methods. To enhance the compliance of the pad, the interdigitated electrodes can be deposited by flexible materials such as carbon mixed with a polymer binder [2] or crumpled metal film [20]. The cover and substrate can be made of elastomers, polymers or plastics with different dielectric constants while the supporting wall can be any common material, e.g. concrete cement or wood.

A four-layer model is here established, including the dielectric wall, the air gap, the cover, and the dielectric substrate. To investigate the electroadhesion, we first need to calculate the electric field distribution generated by the electrodes embedded in the electroadhesive device. It is well known that the electric field $\mathbf{E}$ in the solution space can be readily calculated from the electric potential $\phi$ by

$$
\mathbf{E}=-\nabla \phi,
$$


And that the potential in a dielectric medium satisfies the Laplace equation

$$
\nabla^{2} \phi=0
$$

When the solution domain of the problem is filled with a homogenous material, the electrostatic field can be analytically solved by the conformal mapping technique [21, 22]. However, for a structure made of several different materials as shown in Figure 2(a), the conformal mapping technique fails to solve the problem. Therefore, a point matching method is used here to calculate the electric fields $[9,23]$. In this method, the potential function in each layer is defined by a series expansion in terms of the solutions of the Laplace equation, which are periodic in the direction parallel to the electrodes. For different layers, the coefficients in the series are related to each other and to the electric potentials applied on the electrodes. The boundary conditions in the electrode plane are satisfied at $N$ discrete points, with $N$ being the number of terms in the series expansion [23]. The details of the calculation method are given in the supplemental information (SI) [24].

As shown in Figure 2(a), the electroadhesive device consists of a coplanar array of electrodes whose uniform pitch and width are assumed to be $L=2 b$ and $2 a$, respectively. The electrodes are assumed to have a negligible thickness and to be charged alternatingly by the electric potentials $\Phi$ and $-\Phi$. Due to the periodic feature of the electrodes, only one period of the structure is analyzed. More details of the model and the solution method are given in SI[24]. As shown in Figs. 2(b)-(d), using this four-layer model, we calculate the distributions of electric potential $\phi$ and electric field components $E_{x}$ and $E_{y}$ for one period of the structure with the assumed dielectric and geometric parameters $\varepsilon_{w}=10 \varepsilon_{0}, \varepsilon_{c}=2 \varepsilon_{0}, a / b=0.5$, and $t / b=0.02$, where $\varepsilon_{0}$ denotes the dielectric constant of air, $\varepsilon_{w}$ is the dielectric constant of the wall (supporting substrate), $\varepsilon_{c}$ the dielectric constant of the cover, $t$ is the air gap thickness between the electroadhesion pad and the wall. It can be seen that $E_{y}$ is the dominating component and its intensity in the air gap is much larger than those in the dielectric wall and the cover, yielding a relative larger attractive force between the pad and the wall.

The electrostatic attractive force exerted on the dielectric plate arises from the interaction between the applied non-uniform electric field and the induced conduction and polarization charge [9]. Once we have obtained the electric field, the attractive force can be calculated from the Maxwell stress tensor $T_{i j}$, which is defined as

$$
T_{i j}=\varepsilon\left(E_{i} E_{j}-\frac{1}{2} \delta_{i j}|\mathbf{E}|^{2}\right),
$$


where $\varepsilon$ denotes the electric permittivity and $\delta_{i j}$ the Kronecker delta. The attraction force $F_{N}$ along the normal direction of the dielectric surface is obtained by integrating the Maxwell stress tensor $T_{i j}$ over the enclosing surfaces of the dielectric medium. Assuming the pad surface is parallel to the wall (Figure 2(a)), the electroadhesive force acting on the wall of unit length and width $L$ can be calculated by

$$
F_{N}=\int_{L} f_{y} \mathrm{~d} v=\mathbb{D}_{S} T_{y y} \mathrm{~d} s=\frac{1}{2} \varepsilon_{0} \int_{0}^{L}\left(E_{y}^{2}-E_{x}^{2}\right) \mathrm{d} l,
$$

where $E_{x}$ and $E_{y}$ are the electric field components in the air-gap between the electroadhesive device and the dielectric wall.

Because the wall is regarded to be infinite in length along the $y$-direction, the attraction force acting on the upper surface of the wall is negligible. The electric field is uniform in the $z$-direction when the marginal part of the pad is neglected. Then the electroadhesive force acting on the whole dielectric wall of a period length $L$ is calculated by

$$
F_{N}=\left.\frac{1}{2} \varepsilon_{0} \int_{0}^{L}\left[\left(\varepsilon_{w} / \varepsilon_{0}\right)^{2} E_{1 y}{ }^{2}-E_{1 x}{ }^{2}\right] \mathrm{d} l\right|_{y=h_{2}^{+}},
$$

where $E_{1 x}$ and $E_{1 y}$ are the components of the electric field on the surface of the dielectric wall. Thus, the average adhesion stress can be given by

$$
\sigma_{\mathrm{ad}}=\frac{1}{4 b} \varepsilon_{0} \int_{0}^{2 b}\left[\left(\frac{\varepsilon_{w}}{\varepsilon_{0}}\right)^{2} E_{1 y}{ }^{2}-E_{1 x}{ }^{2}\right] \mathrm{d} x
$$

With the assumption of the electric potential $\phi_{1}$ for the infinite wall, as shown in the SI[24], one can prove

$$
\begin{aligned}
\left.\int_{0}^{L} E_{1 y}{ }^{2} \mathrm{~d} x\right|_{y=h_{2}^{+}}=\left.\int_{0}^{L} E_{1 x}{ }^{2} \mathrm{~d} x\right|_{y=h_{2}^{+}} & \text {Thus, Eq. (6) becomes } \\
\sigma_{\text {ad }} & =\frac{1}{2} \varepsilon_{0}\left[\left(\frac{\varepsilon_{w}}{\varepsilon_{0}}\right)^{2}-1\right] C\left(\frac{a}{b}, \frac{h_{1}}{b}, \frac{t}{b}, \frac{\varepsilon_{c}}{\varepsilon_{w}}\right)\left(\frac{\Phi}{2 b}\right)^{2},
\end{aligned}
$$

where $C\left(\frac{a}{b}, \frac{h_{1}}{b}, \frac{t}{b}, \frac{\varepsilon_{c}}{\varepsilon_{w}}\right)$ is defined as a dimensionless function of geometric parameters $\frac{a}{b}, \frac{h_{1}}{b}$ and $\frac{t}{b}$, and material parameter $\frac{\varepsilon_{c}}{\varepsilon_{w}} \cdot\left(\frac{\Phi}{2 b}\right)^{2}$ is the normalized electric field generated by the applied voltage. The factor $\frac{1}{2} \varepsilon_{0}\left[\left(\frac{\varepsilon_{w}}{\varepsilon_{0}}\right)^{2}-1\right]$ is a constant depending only on the dielectric constants of the air and the wall. It is seen from Eq. (7) that the 
electroadhesive force is proportional to the square of the applied voltage [15] when the function $C\left(\frac{a}{b}, \frac{h_{1}}{b}, \frac{t}{b}, \frac{\varepsilon_{w}}{\varepsilon_{c}}\right)$ and the dielectric constant of the wall $\varepsilon_{w}$ are fixed. In the following section, we will use Eq. (7) to examine the effect of geometric and material parameters on the electroadhesive force generated by the electroadhesive pad.

\section{Results and Discussions}

\subsection{Electroadhesion without air-gap at the interface}

We first examine the special case, in which the electroadhesion pad contacts the supporting wall without the airgap, i.e., there exists a zero-thickness air-gap between the contact surface of electroadhesion pad and the wall. The dielectric wall and the cover of the pad usually have different dielectric constants, i.e., $\varepsilon_{c} \neq \varepsilon_{w}$. Since no analytical solution can be obtained for such an inhomogeneous medium, the point matching method is employed to calculate the electric field with the four-layer model presented in Section II. With the zero-thickness air-gap and $\varepsilon_{c} \neq \varepsilon_{w}$, Eq. (7) can be re-expressed as

$$
\sigma_{\text {ad }}=\frac{1}{2} \varepsilon_{0}\left[\left(\frac{\varepsilon_{w}}{\varepsilon_{0}}\right)^{2}-1\right] C^{\prime}\left(\frac{a}{b}, \frac{h_{1}}{b}, \frac{\varepsilon_{c}}{\varepsilon_{w}}\right)\left(\frac{\Phi}{2 b}\right)^{2},
$$

where $C^{\prime}\left(a / b, h_{1} / b, \varepsilon_{c} / \varepsilon_{w}\right)$ is a newly defined function of geometric parameter $a / b, h_{1} / b$ and material parameter $\varepsilon_{c} / \varepsilon_{w}$.

Figs. 3(a) and (b) plot the variation of $C^{\prime}\left(a / b, h_{1} / b, \varepsilon_{c} / \varepsilon_{w}\right)$ with respect to the ratio of dielectric constants $\varepsilon_{c} / \varepsilon_{w}$ for various $h_{1} / b$ and $a / b$, respectively. It can be seen from Figure 3 that $C^{\prime}\left(a / b, h_{1} / b, \varepsilon_{c} / \varepsilon_{w}\right)$ increases with the increase in $\varepsilon_{c} / \varepsilon_{w}$ for a fixed value of $h_{1} / b$. However, the influence of $\varepsilon_{c} / \varepsilon_{w}$ becomes less significant when $\varepsilon_{c} / \varepsilon_{w}>5$, and thus each curve has a plateau. As a result, from the viewpoint of fabrication, it is desired to select materials with higher dielectric constants as the cover. This inspires us to select proper devices in various working environments [2]. It is also found that the dielectric constant of the substrate material has no influence on the electroadhesive performance of the device. In addition, Figure 3 shows that both the normalized electrode width $a / b$ and the cover thickness $h_{1} / b$ have significant effects on electroadhesion, and that a smaller $h_{1} / b$ or a larger $a / b$ results in a larger electrostatic attractive force on the supporting wall. These results indicate that it is better to reduce the cover thickness and to increase the electrode width in order to enhance the adhesion force of the 
electroadhesive device. However, it should be noticed that the electrodes must not reach the value $a / b=1$, at which the positive and negative electrodes would touch each other to form a short circuit. In the above discussions, we have ignored the restrictions of dielectric materials and allow the maximum voltage to be increased as required. However, the applied voltage cannot be increased infinitely due to the breakdown voltage of dielectric materials. For electroadhesion, this limit is determined by the breakdown electric strength of air $E_{B D}^{a i r}$, which is about $3 \times 10^{6} \mathrm{~V} / \mathrm{m}$.

Therefore, when the applied voltage reaches the threshold of breakdown, there is an optimal value for $a / b$ to maximize the electroadhesive force (See supplemental material SI.3 [24]). To validate the above theoretical model, we calculate the electroadhesion force generated by an electroadhesive pad described in the literature [25], which has the same structural configuration as shown in Figure 1(b). In this design, the cover layer of the pad is $123 \mu \mathrm{m}$ in thickness, made of epoxy resin (dielectric constant $\varepsilon_{c}=7.8$ ), and the substrate is made of glass-epoxy resin. The copper electrodes are $35 \mu \mathrm{m}$ thick and $1 \mathrm{~mm}$ wide. To measure the electroadhesion generated by the pad, a 4-inch silicon wafer was used as the supporting wall. As shown in Figure 3(c), the theoretical results agree with the experimental data. The little discrepancy may result from the fact that in the ideal model the electrodes are considered to have zero thickness and that there existed fringe effect in the testing device.

\subsection{Electroadhesion with an air gap at the interface}

In the former section, we discussed the ideal case with perfect contact between the electroadhesive device and the supporting wall. In practice, however, a small air-gap with a thickness of just a few hundred nanometers exists between the electroadhesive pad and the wall [18]. Thus, it is necessary to investigate the effect of the air-gap thickness on the electroadhesive force. Figure $4\left(\right.$ a) shows the variation of $C\left(\frac{a}{b}, \frac{h_{1}}{b}, \frac{t}{b}, \frac{\varepsilon_{c}}{\varepsilon_{w}}\right)$ as a function of normalized air-gap $t / b$ when $h_{1} / b=0.02, \varepsilon_{c}=7 \varepsilon_{0}$ and $\varepsilon_{w}=5 \varepsilon_{0}$ for different values of the electrode width $a / b$. It can be seen that the air-gap indeed has a significant effect on electroadhesion, and a very small air-gap (e.g., $0.05 b$ ) can drastically reduce about by $70 \%$ of its original force when $a / b=0.8$. It is also noted that the degeneration of the attractive force is relatively faster for a smaller thickness of the cover. For some representative values of the air-gap $t / b$ in the range from 0 to 0.2 , Figure $4(\mathrm{~b})$ shows the variation of $C\left(\frac{a}{b}, \frac{h_{1}}{b}, \frac{t}{b}, \frac{\varepsilon_{c}}{\varepsilon_{w}}\right)$ as a function of the electrode width $a / b$ when $h_{1} / b=0.02, \varepsilon_{c}=7 \varepsilon_{0}$ and $\varepsilon_{w}=5 \varepsilon_{0}$. It is found that the attractive force 
increases with the increase in the electrode width $a / b$, but the influence of $a / b$ sharply reduces when the air-gap $t / b$ is large. Thus it is concluded that the reducing air-gap thickness is important to increase the electroadhesive force.

\subsection{Electroadhesion on a wall with finite thickness}

In the above analyses, for the sake of simplicity, we assumed that the supporting wall is of infinite thickness compared with the electroadhesive pad. However, in such practical applications as electrostatic-levitation, we often encounter objects with finite thickness, e.g., thin LCD panels. Therefore, it is essential to understand the influence of the thickness of targeting substrates (i.e., attachments, walls or panels). In what follows, we employ a five-layer model (For details of the model, see supplemental materials SI.2[24]) to study the effect of the thickness of supporting structure or attachment (e.g., wall). The electroadhesive force acting on the whole dielectric wall of a period length $L$ is calculated by

$$
F_{N}=\frac{1}{2} \varepsilon_{0} \int_{0}^{L}\left[\left(E_{3 y}{ }^{2}-E_{3 x}{ }^{2}\right)-\left(E_{1 y}{ }^{2}-E_{1 x}{ }^{2}\right)\right] \mathrm{d} l,
$$

where $E_{1 x}$ and $E_{1 y}$ are the electric field components above the dielectric wall, and $E_{3 x}$ and $E_{3 y}$ are the electric field components below the wall (See Figure S1 in supplemental material[24]). The average adhesion stress is written as

$$
\sigma_{\mathrm{ad}}=\varepsilon_{0} \bar{C}\left(\frac{a}{b}, \frac{h_{1}}{b}, \frac{t}{b}, \frac{\varepsilon_{c}}{\varepsilon_{w}}\right)\left(\frac{\Phi}{2 b}\right)^{2}
$$

where $\bar{C}\left(\frac{a}{b}, \frac{h_{1}}{b}, \frac{t}{b}, \frac{\varepsilon_{c}}{\varepsilon_{w}}\right)$ is a defined dimensionless factor. Figure 5(a) describes the variation of $\bar{C}\left(\frac{a}{b}, \frac{h_{1}}{b}, \frac{t}{b}, \frac{\varepsilon_{c}}{\varepsilon_{w}}\right)$ with the normalized wall thickness $H / b$. For each distinct $\varepsilon_{w} / \varepsilon_{c}$, we fix the electrode width as $a / b=0.8$ and $h_{1} / b=0.02$, and assume there is no air-gap at the contact interface. It can be found from Figure 5 that finite wall thickness has a significant effect on electroadhesion. For a given value of $\varepsilon_{w} / \varepsilon_{c}$, the electroadhesion stress increases with wall thickness, and the stress approaches a limit value when the wall thickness exceeds $2 b$. For different air-gaps, Figure 5(b) shows the variation of $\bar{C}\left(\frac{a}{b}, \frac{h_{1}}{b}, \frac{t}{b}, \frac{\varepsilon_{c}}{\varepsilon_{w}}\right)$ with respect to the normalized wall thickness 
$H / b$, where we take the parameters $a / b=0.8, h_{1} / b=0.02, \varepsilon_{c}=5$, and $\varepsilon_{w}=7.5$. It can be seen that the wall thickness has more significant impact when the air-gap is smaller.

\subsection{Effect of surface roughness}

In Section III.B, we have shown that the electro-attractive force is significantly affected by the air-gap between pad and wall. Thus, the electroadhesive force of a rigid pad will be sensitive to its surface roughness. Therefore, one possible approach to alleviate the negative influence of roughness is to develop a compliant pad, e.g., depositing compliant electrodes into elastomeric insulators, which is able to adaptively adhere to a wide range of roughness [15]. Another promising method is to design a compliant electrostatic pad by mimicking the structure of gecko feet [26-28]. Such a biomimetic structure enables the electroadhesive pad to maintain a much larger electroadhesive force on rough surfaces. To design an adaptive electroadhesion pad like gecko feet, one can add a layer consisting of short hairs on top of the cover layer of the pad to enhance its adaptability on rough surfaces, as shown in Figure 6(a). To analyze the performance of the biomimetic compliant pad using the present model, the added layer can be treated as a composite with an effective dielectric constant $\varepsilon_{m}$, which can be evaluated by a parallel mixing rule[29] as $\varepsilon_{m}=V_{a} \varepsilon_{c}+V_{a} \varepsilon_{0}$, where $V_{a}$ and $V_{c}$ are the volume fractions of the dielectric phase and air-gap phase $\left(V_{a}+V_{c}=1\right)$,

respectively. For different values of the hair length, Figure 6(b) shows the variation of $\overline{\bar{C}}\left(\frac{a}{b}, \frac{h_{1}}{b}, \frac{t}{b}, \frac{\varepsilon_{c}}{\varepsilon_{w}}, \frac{\varepsilon_{m}}{\varepsilon_{w}}, \frac{h_{m}}{b}\right)$ as a function of dielectric constants ratio $\varepsilon_{m} / \varepsilon_{w}$ where we take $a / b=0.9, h_{1} / b=0.01, t / b=0, \varepsilon_{w}=5 \varepsilon_{0}$, and $\varepsilon_{c}=7 \varepsilon_{0}$. It is obvious that the electroadhesion reduces as the length of hair layer increases and will reach a peak value for each $h_{m} / b$ when the mixed dielectric constant $\varepsilon_{m}$ is larger than $\varepsilon_{w}$. It is expected that such a design has another attractive merit, i.e., combining the electroadhesive force together with the van der Waals force to generate stronger adhesion. It is known that when the dimension of gecko-feet-like structures goes down to the micro or even nano scale, the van der Waals force generated by the hair layer becomes much stronger[26-28, 30, 31]. Therefore, the hair layer not only improves the adaptability of a pad on rough surfaces but also enhances the attraction force. It will be promising to fabricate a novel electroadhesive device or to develop a new micro-transfer technique by superimposing both electroadhesive and van der Waals forces.

\section{CONCLUSIONS}


In summary, we have theoretically analyzed the electroadhesive forces of an interdigitated type electroadhesive device by employing a semi-analytical model and discussed the optimal design principles of electroadhesive devices for a wide variety of applications. It is found that, to enhance the electroadhesive force, the cover layer should employ dielectric materials with larger dielectric constants, and the electrodes should be wider while the cover should be thinner. However, for the extreme case where the electric potential is equal to the breakdown voltage, the maximum electroadhesive force will occur with a very small ratio of $a / b$. We have also proposed a novel route to improve the performance of electroadhesive pads by mimicking the structure of gecko feet, which enables the electrodes to maintain a good proximity with rough surfaces and to combine both electroadhesion and van der Waals forces together to increase the overall adhesive force. The present theoretical model is helpful to design new electroadhesive devices and to develop electroadhesion-based micro-transfer techniques.

\section{ACKNOWLEDGMENTS}

The work was financially supported by Australian National University, Duke University and the NSF's Research Triangle Materials Research Science and Engineering Center (DMR-1121107). C.C. would like to thank Prof. Xuanhe Zhao from MIT for helpful discussion and support. Q.Q. thanks the financial support from the Australian Research Council. X.F. acknowledges the 973 Program of MOST (2012CB934001).

\section{REFERENCES}

[1] Berengueres J, Urago M, Saito S, Tadakuma K, Meguro H. Gecko inspired Electrostatic Chuck. Robotics and Biomimetics, 2006 ROBIO '06 IEEE International Conference on. 2006:1018-23.

[2] Prahlad H, Pelrine R, Stanford S, Marlow J, Kornbluh R. Electroadhesive robots-wall climbing robots enabled by a novel, robust, and electrically controllable adhesion technology. Robotics and Automation, 2008 ICRA 2008 IEEE International Conference on. 2008:3028-33.

[3] Vankov AB, Huie P, Blumenkranz MS, Palanker DV. Electroadhesive forceps for tissue manipulation. Proc SPIE 5314, Ophthalmic Technologies XIV. 2004;5314:270-4.

[4] Jeon JU, Higuchi T. Electrostatic suspension of dielectrics. IEEE Transactions on Industrial Electronics. 1998;45:938-46.

[5] Murphy MP, Kute C, Mengüç Y, Sitti M. Waalbot II: Adhesion recovery and improved performance of a climbing robot using fibrillar adhesives. International Journal of Robotics Research. 2011;30:118-33.

[6] Noohi E, Mahdavi SS, Baghani A, Ahmadabadi MN. Wheel-based climbing robot: Modeling and control. Advanced Robotics. 2010;24:1313-43.

[7] Longo D, Muscato G, Tarantello G. Performance Evaluation of Electrostatic Adhesion for Climbing Robots. Proceedings of the 13th International Conference on Climbing and Walking Robots and the Support Technologies for Mobile Machines. 2010:1029-36.

[8] Shao Ju W, Jeon J-U, Higuchi T, Ju J. Electrostatic force analysis of electrostatic levitation system. SICE '95 Proceedings of the 34th SICE Annual Conference International Session Papers. 1995:1347-52. 
[9] Woo S, Higuchi T. Electric field and force modeling for electrostatic levitation of lossy dielectric plates. Journal of Applied Physics. 2010;108:104906--10.

[10] Jin L, Higuchi T, Kanemoto M. Electrostatic levitator for hard disk media. IEEE Transactions on Industrial Electronics. 1995;42:467-73.

[11] Asano K, Hatakeyama F, Yatsuzuka K. Fundamental study of an electrostatic chuck for silicon wafer handling. Industry Applications, IEEE Transactions on. 2002;38:840-5.

[12] Kanno S, Usui T. Generation mechanism of residual clamping force in a bipolar electrostatic chuck. Journal of Vacuum Science \& Technology B: Microelectronics and Nanometer Structures. 2003;21:2371-7.

[13] Monkman GJ. Robot grippers for use with fibrous materials. International Journal of Robotics Research. 1995;14:144-51.

[14] Monkman G. Electroadhesive microgrippers. Industrial Robot: An International Journal. 2003;30:326-30.

[15] Yamamoto A, Nakashima T, Higuchi T. Wall Climbing Mechanisms Using Electrostatic Attraction Generated by Flexible Electrodes. Micro-NanoMechatronics and Human Science, 2007 MHS '07 International Symposium on. 2007:389-94.

[16] Murphy MP, Sitti M. Waalbot: agile climbing with synthetic fibrillar dry adhesives. Proceedings of the 2009 IEEE international conference on Robotics and Automation. Kobe, Japan: IEEE Press; 2009. p. 2637-8.

[17] Ltd. JI. http://www.justick.com/Products.aspx. 2006.

[18] Yoo J, Choi JS, Hong SJ, Kim TH, Lee SJ. Finite element analysis of the attractive force on a Coulomb type electrostatic chuck. International Conference on Electrical Machines and Systems. 2007:1371-5.

[19] Kanno S, Usui T. Generation mechanism of residual clamping force in a bipolar electrostatic chuck. Journal of Vacuum Science \& Technology B. 2003;21:2371-7.

[20] Cao C, Chan HF, Zang J, Leong KW, Zhao X. Harnessing Localized Ridges for High-Aspect-Ratio Hierarchical Patterns with Dynamic Tunability and Multifunctionality. Advanced Materials. 2014;26:1763-70.

[21] Lim YC, Moore RA. Properties of alternately charged coplanar parallel strips by conformal mappings. IEEE Transactions on Electron Devices. 1968;15:173-80.

[22] Wheeler HA. Transmission-Line Properties of Parallel Wide Strips by a Conformal-Mapping Approximation. Microwave Theory and Techniques, IEEE Transactions on. 1964;12:280-9.

[23] Marcuse D. Electrostatic field of coplanar lines computed with the point matching method. IEEE Journal of Quantum Electronics. 1989;25:939-47.

[24] See supplemental materials as [] for the detailed formulations of four-later model and five layer model 2014.

[25] Yatsuzuka K, Hatakeyama F, Asano K, Aonuma S. Fundamental characteristics of electrostatic wafer chuck with insulating sealant. Industry Applications, IEEE Transactions on. 2000;36:510-6.

[26] Gao H, Wang X, Yao H, Gorb S, Arzt E. Mechanics of hierarchical adhesion structures of geckos. Mechanics of Materials. 2005;37:275-85.

[27] Yao H, Gao H. Mechanics of robust and releasable adhesion in biology: Bottom-up designed hierarchical structures of gecko. Journal of the Mechanics and Physics of Solids. 2006;54:1120-46.

[28] Autumn K, Sitti M, Liang YA, Peattie AM, Hansen WR, Sponberg S, et al. Evidence for van der Waals adhesion in gecko setae. Proceedings of the National Academy of Sciences. 2002;99:12252-6.

[29] Wu Y, Zhao X, Li F, Fan Z. Evaluation of mixing rules for dielectric constants of composite dielectrics by MCFEM calculation on 3D cubic lattice. Journal of electroceramics. 2003;11:227-39.

[30] Lee H, Lee BP, Messersmith PB. A reversible wet/dry adhesive inspired by mussels and geckos. Nature. 2007;448:338-41.

[31] Autumn K, Gravish N. Gecko adhesion: evolutionary nanotechnology. Philosophical Transactions of the Royal Society A: Mathematical, Physical and Engineering Sciences. 2008;366:1575-90. 


\section{Figure Captions}

Figure 1. Schematic of an electroadhesive pad with embedded interdigitated electrodes: (a) 3D view and (b) side view.

Figure 2. (a) A four-layer model for an electroadhesive pad with periodic coplanar electrodes sandwiched between a dielectric cover and a substrate. Contour plots of the calculated (b) electric potential $\phi$, (c) electric field component $E_{x}$, and (d) electric field component $E_{y}$ for a period of the model in (a).

Figure 3. Variation of $C^{\prime}\left(a / b, h_{1} / b, \varepsilon_{c} / \varepsilon_{w}\right)$ as a function of dielectric constant ratio $\varepsilon_{c} / \varepsilon_{w}$ (a) for various normalized cover thickness $h_{l} / b$ when $a / b=0.8$ and $\varepsilon_{w}=5 \varepsilon_{0}$; and (b) for various normalized electrode width $a / b$ when $h_{1} / b=0.02$ and $\varepsilon_{w}=5 \varepsilon_{0}$. (c) Comparison of the predicted electroadhesion with the experimental data from literature.

Figure 4. (a) Variation of $C\left(a / b, h_{1} / b, t / b, \varepsilon_{c} / \varepsilon_{w}\right)$ as a function of dielectric constants ratio $t / b$ for various normalized electrode width $a / b$ when $h_{1} / b=0.02, \varepsilon_{c}=7 \varepsilon_{0}$, and $\varepsilon_{w}=5 \varepsilon_{0}$. (b) Variation of $C\left(a / b, h_{1} / b, t / b, \varepsilon_{c} / \varepsilon_{w}\right)$ as a function of dielectric constants ratio $a / b$ for various normalized thickness of air-gap $t / b$ when $h_{l} / b=0.02$, $\varepsilon_{c}=7 \varepsilon_{0}$, and $\varepsilon_{w}=5 \varepsilon_{0}$.

Figure 5. (a) Variation of $\bar{C}\left(a / b, h_{1} / b, t / b, \varepsilon_{c} / \varepsilon_{w}\right)$ as a function of normalized wall thickness $H / b$ for different dielectric constants ratio $\varepsilon_{c} / \varepsilon_{w}$ when $a / b=0.8, h_{l} / b=0.02, t=0$. (b) Variation of $\bar{C}\left(a / b, h_{1} / b, t / b, \varepsilon_{c} / \varepsilon_{w}\right)$ as a function of normalized wall thickness $H / b$ for different air-gap $\mathrm{t} / \mathrm{b}$ when $\mathrm{a} / \mathrm{b}=0.8, h_{l} / b=0.02, \varepsilon_{c}=5 \varepsilon_{0}$ and $\varepsilon_{w}=7.5 \varepsilon_{0}$.

Figure 6. (a) Schematic design of a compliant electroadhesive pad with added hair layer by mimicking Gecko feet. (b) Variation of $\overline{\bar{C}}\left(a / b, h_{1} / b, t / b, \varepsilon_{c} / \varepsilon_{w}, \varepsilon_{m} / \varepsilon_{w}, h_{m} / b\right)$ as a function of dielectric constants ratio $\varepsilon_{m} / \varepsilon_{w}$ for different hair length $h_{m} / b$ when $a / b=0.9, h_{l} / b=0.01, t=0$, where $\varepsilon_{c}=7 \varepsilon_{0}$ and $\varepsilon_{w}=5 \varepsilon_{0}$. 
Figures

(a)

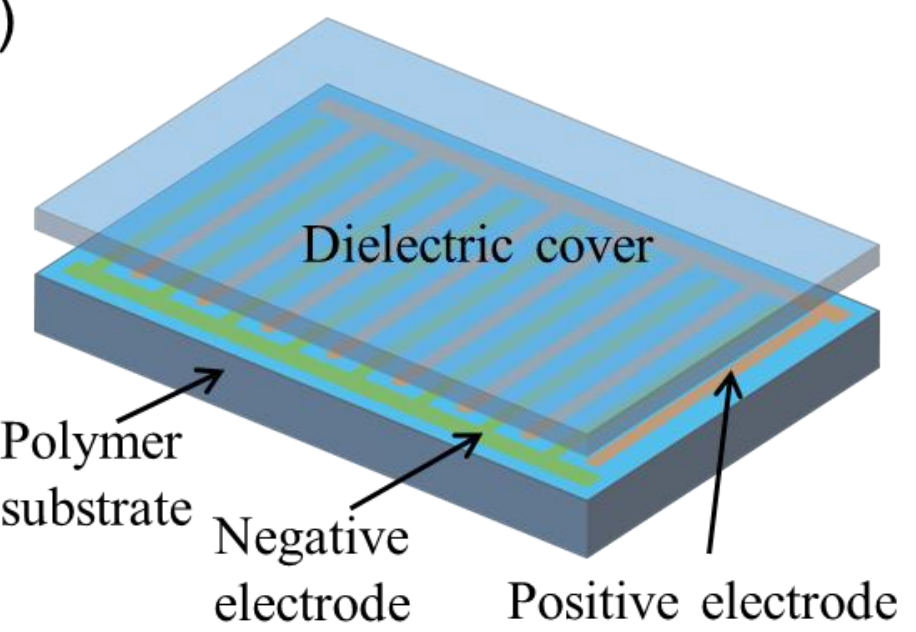

(b)

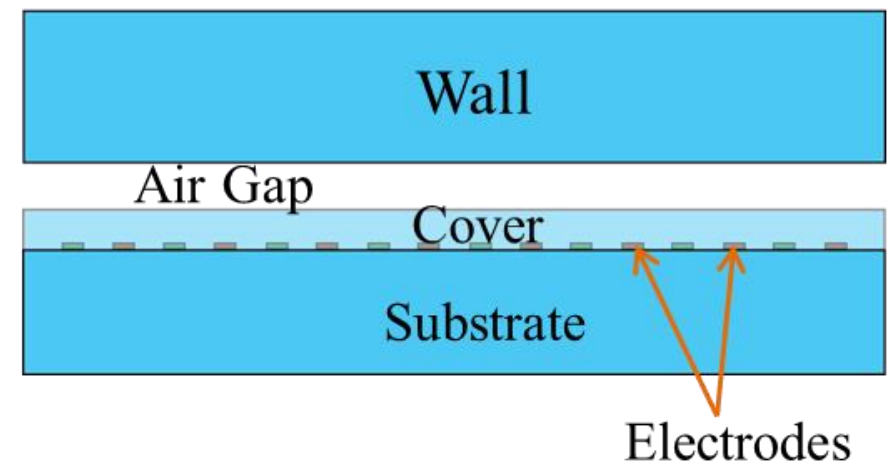


(a)

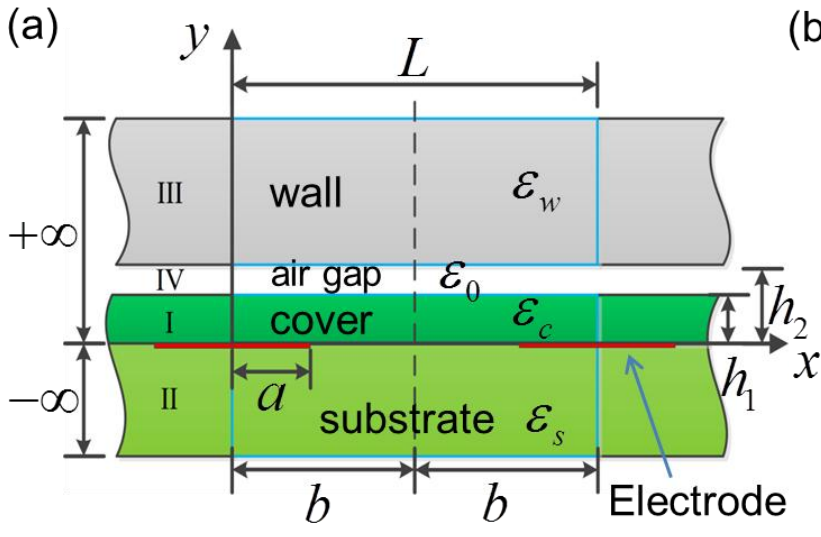

(c)

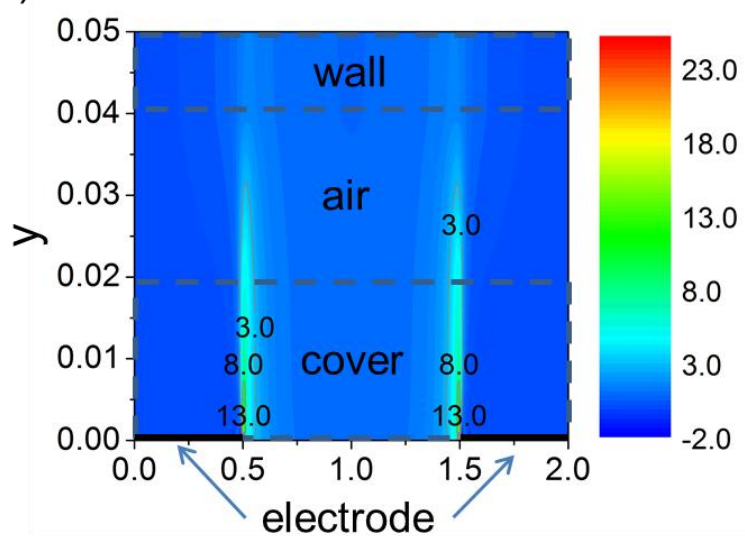

(b)

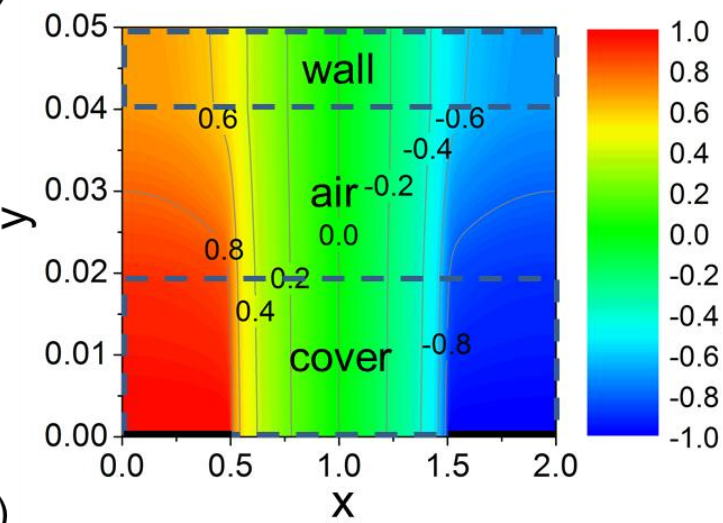

(d)

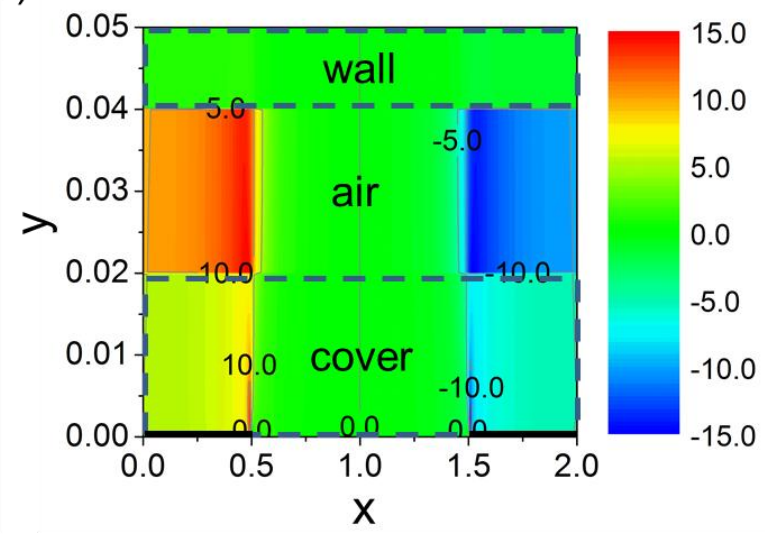




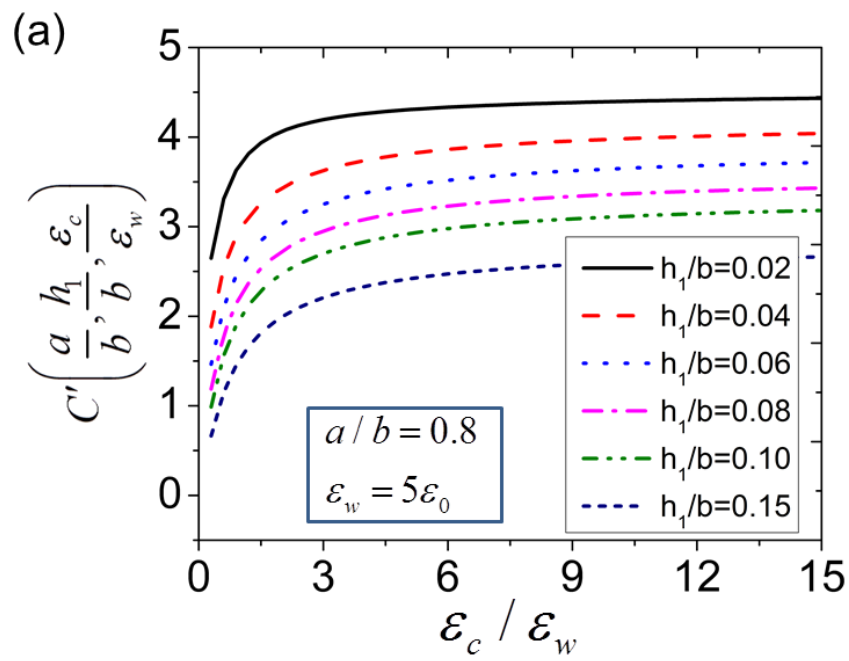

(b)

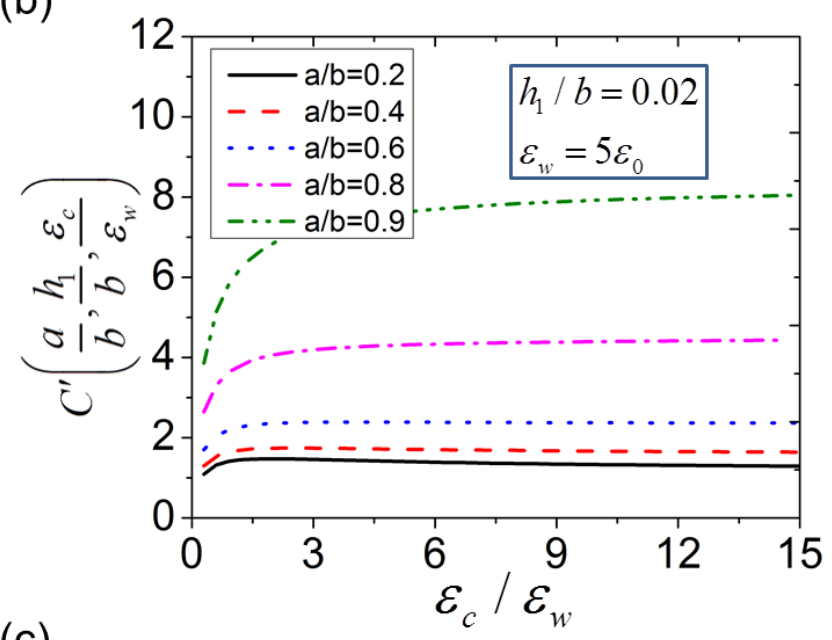

(c)

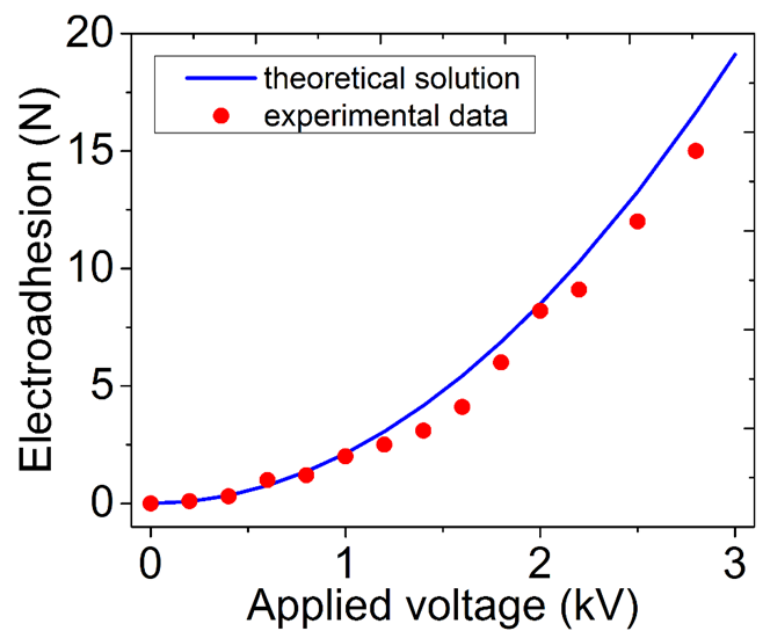


(a)

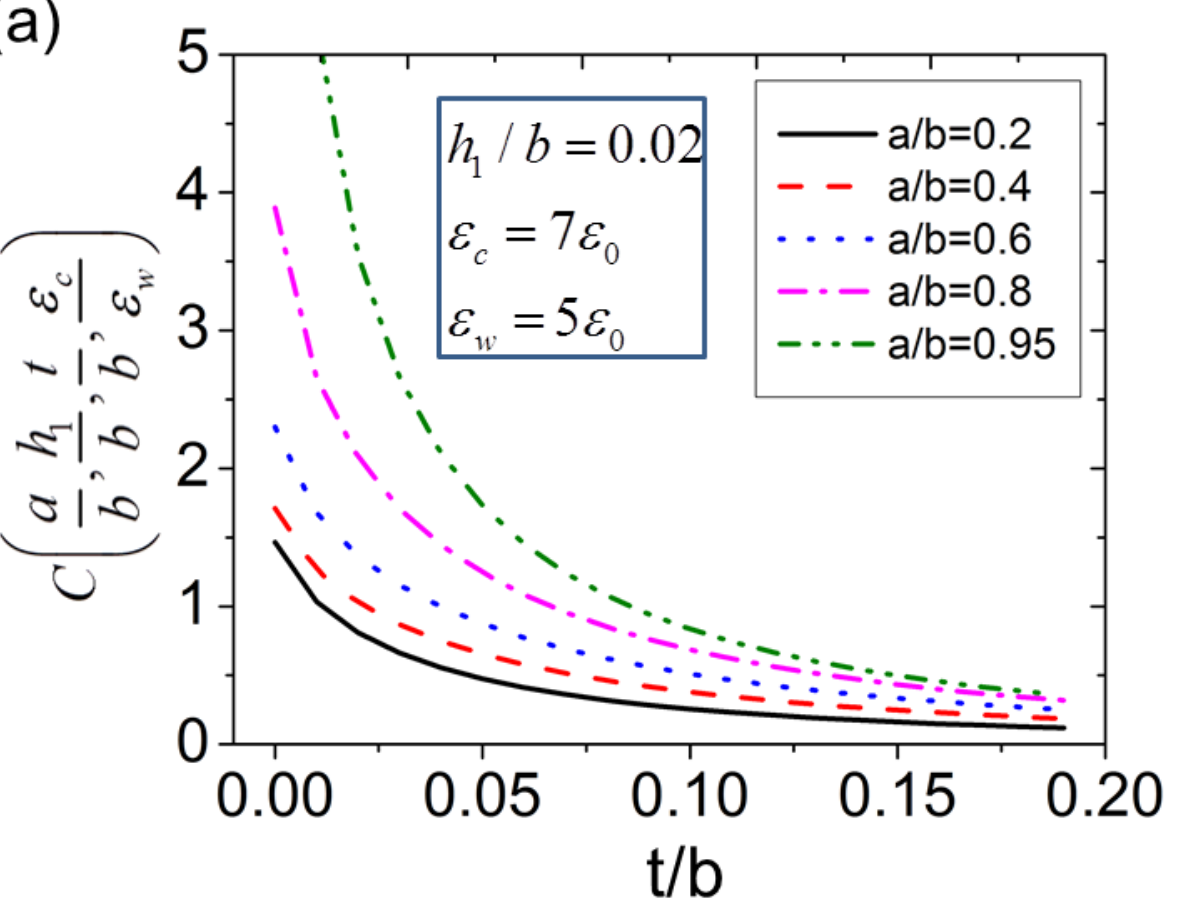

(b)

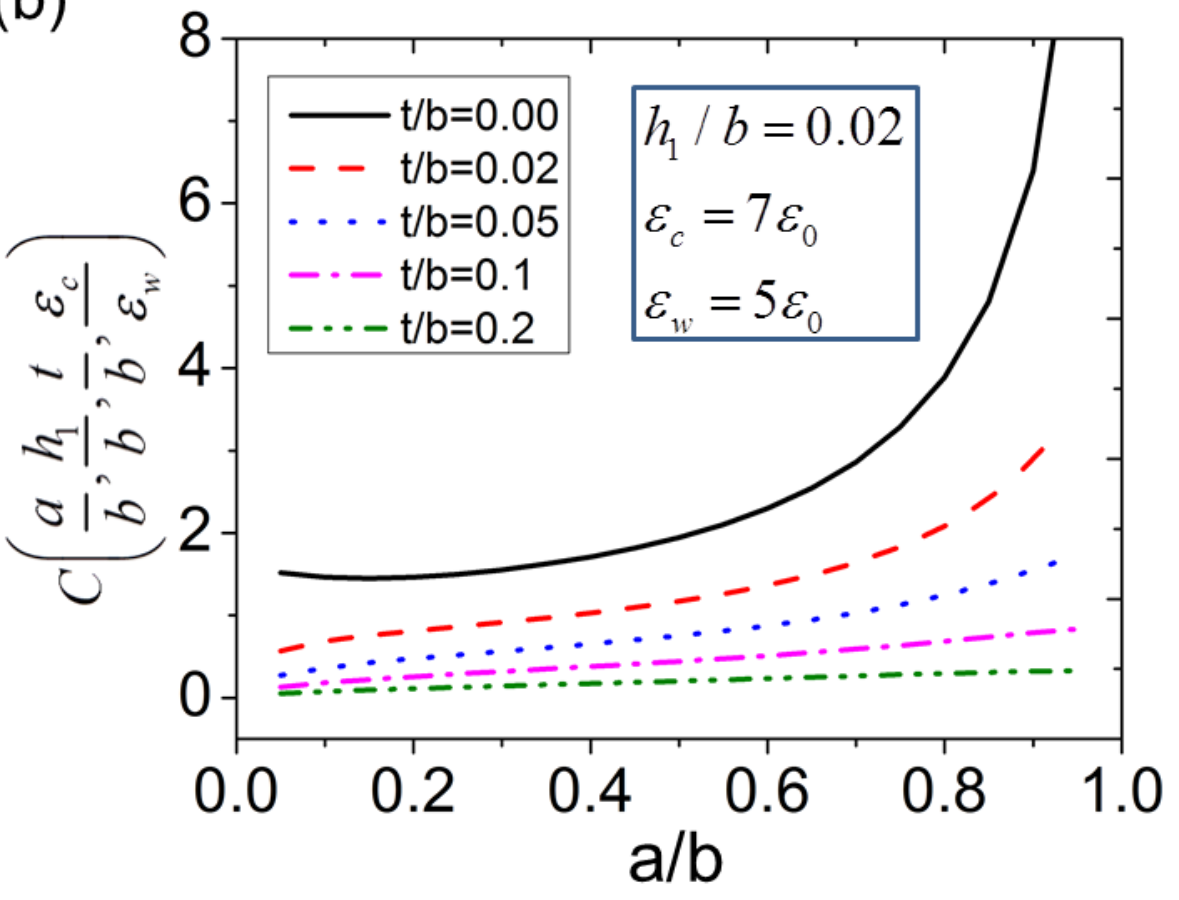


(a)

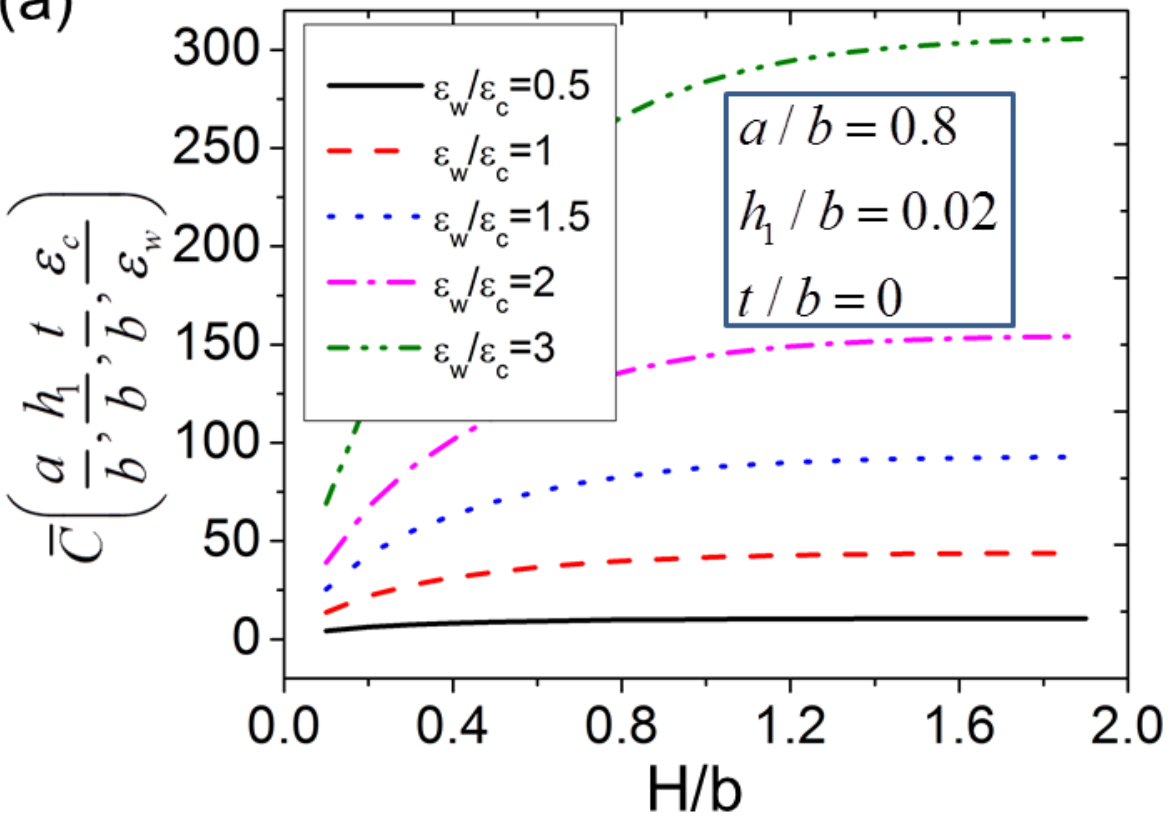

(b)

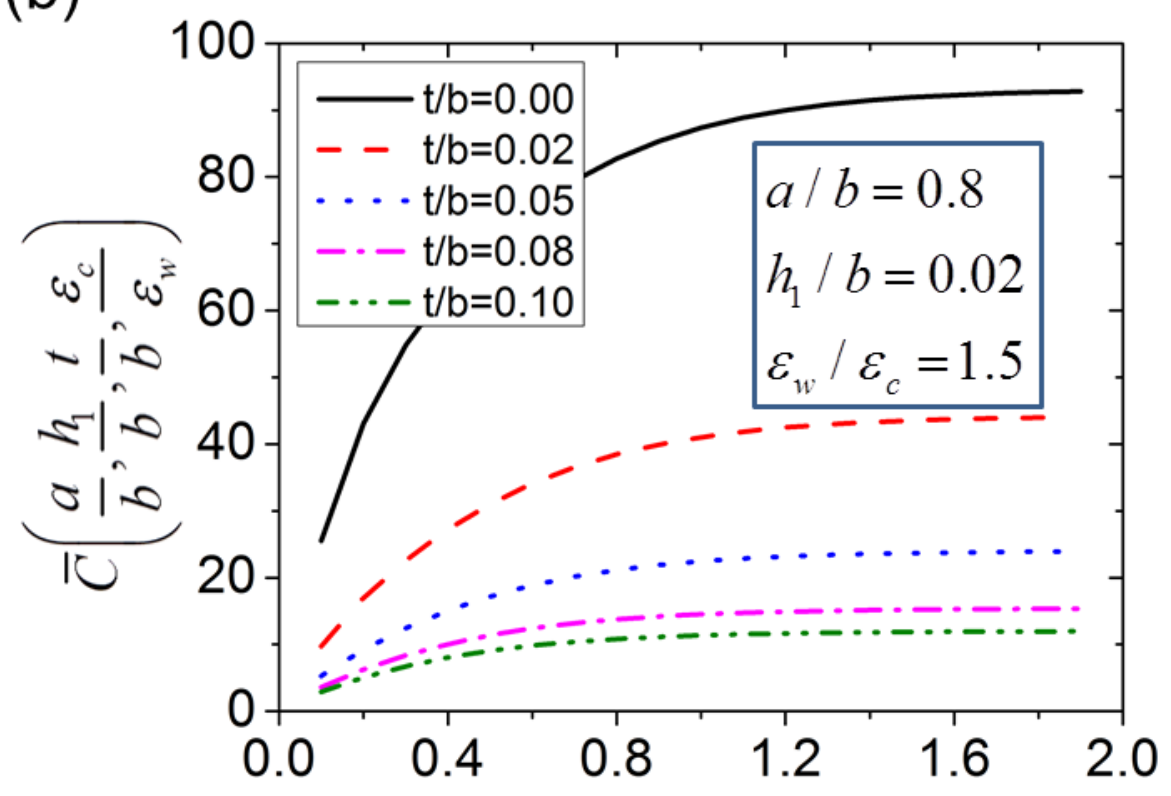


(a)

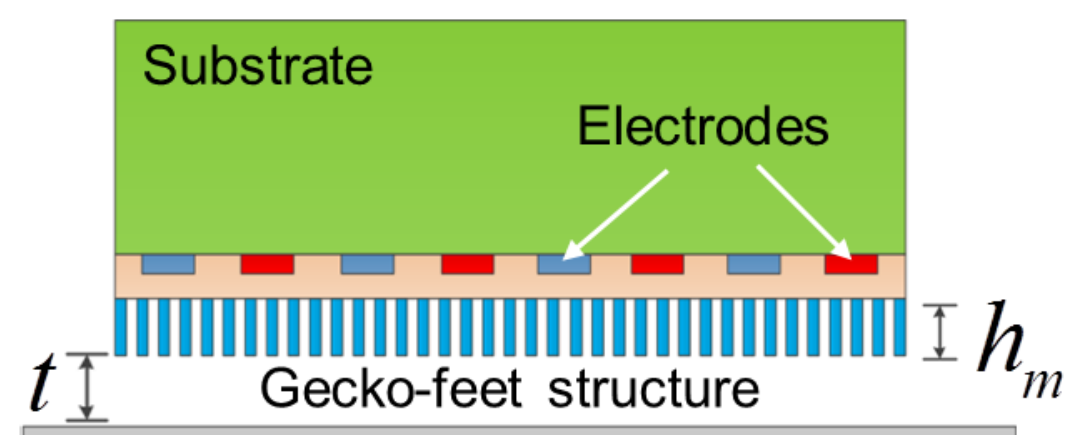

Supporting wall

(b)

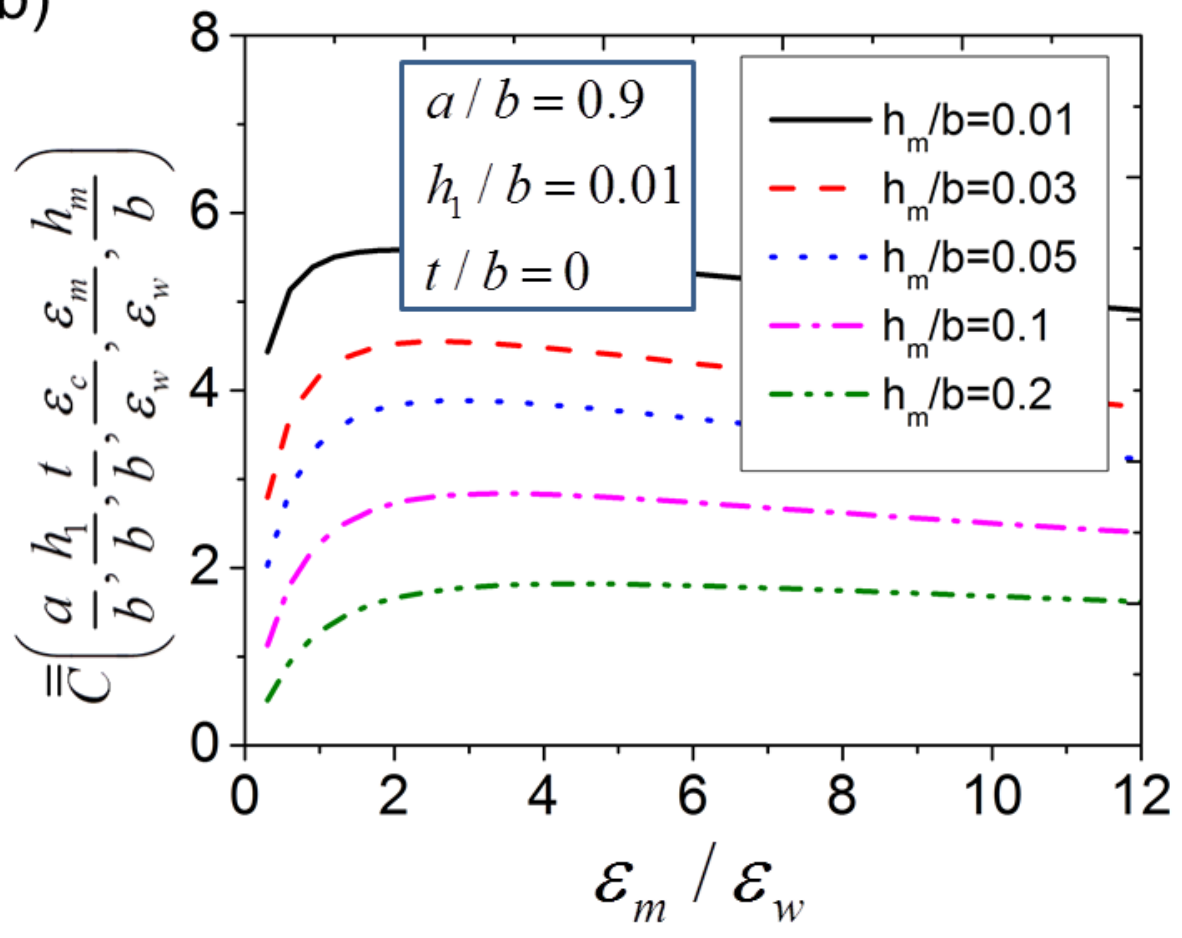




\section{Graphical Abstract:}
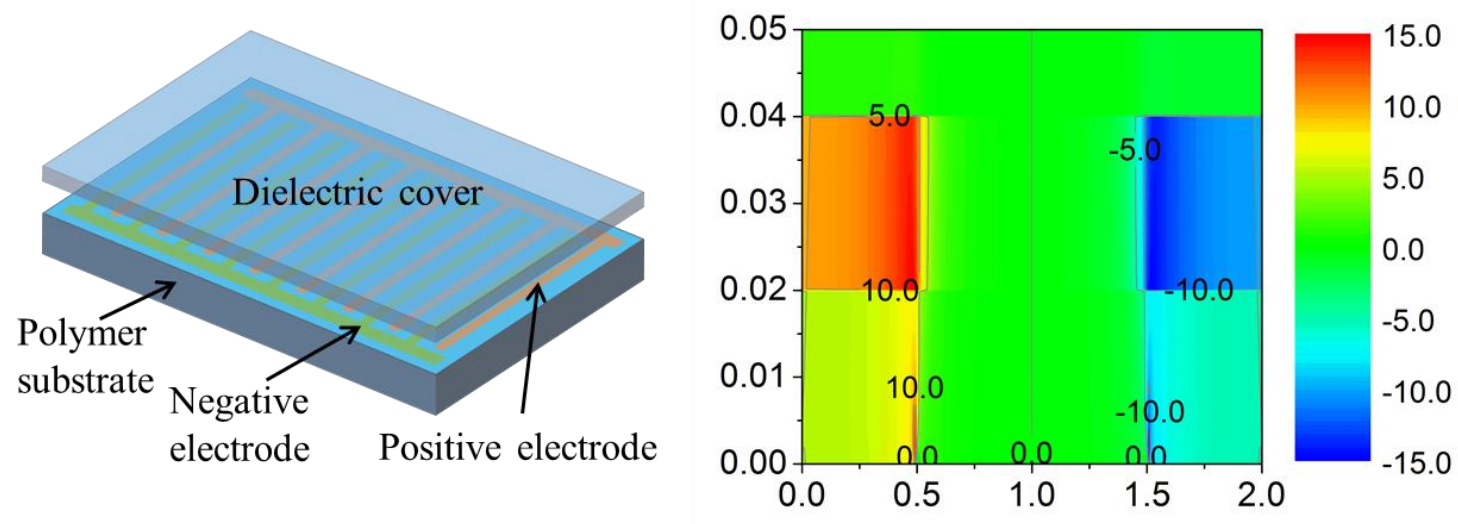